\title{
Rising HIV prevalence among men who have sex with men in Nigeria: a trend analysis
}

George I.E Eluwa ${ }^{1 *} \mathbb{D}$, Sylvia B. Adebajo ${ }^{1}$, Titilope Eluwa², Obinna Ogbanufe ${ }^{3}$, Oluwafunke llesanmi ${ }^{4}$ and Charles Nzelu ${ }^{5}$

\begin{abstract}
Background: Men who have sex with men (MSM) are conservatively estimated to be less than $1 \%$ of the Nigerian population yet nationally account for about $20 \%$ of new HIV infection. We estimated the trend in HIV prevalence and determined correlates of HIV infection among MSM.

Methods: This study used data from respondent-driven sampling in three rounds of integrated biological and behavioral surveillance survey (2007, 2010 and 2014) and covered three states in 2007, six states in 2010 and eight states in 2014. Each round used similar methodology and thus allows for comparison. Behavioral data were obtained using a structured pre-coded questionnaire. Differences in categorical variables were assessed with Chi Square. Logistic regression was used to identify factors associated with HIV.

Results: A total of 879, 1545 and 3611 MSM were recruited in 2007, 2010 and 2014 respectively. Median age was 22 years for 2007 and 2014 while it was 24 years in 2010. About one-third of MSM in 2007 and 2014 and about two-fifths in 2010 had engaged in transactional sex. HIV prevalence increased from 14\% in 2007 to $17 \%$ in 2010 to $23 \%$ in $2014(p<0.0001)$. Factors associated with HIV include older age $\geq 25$ years (adjusted odds ratio \{AOR\}:2.41; 95\% Cl:1.84-3.16); receptive anal sex (AOR:1.92; 95\% Cl:1.54-2.40) and history of sexually transmitted infections (AOR:1.26; 95\% Cl:1.02-1.55).

Conclusion: There's been a consistent and significant increase in HIV prevalence among MSM with about 10percentage points relative increase per year over 7 years. Older MSM were more likely to be HIV positive and this may reflect their prolonged exposure to high risk sexual activities. Evidence based interventions are urgently needed to mitigate intra-group HIV transmission and propagation of HIV epidemic between MSM and the general population.
\end{abstract}

Keywords: Men who have sex with men, MSM, HIV, Trend analysis, Nigeria

\section{Background}

Globally, men who have sex with men (MSM) remain disproportionately infected and affected by $\operatorname{HIV}[1,2]$. Despite huge investments in global HIV and expanded antiretroviral treatment (ART) programs that have resulted in significant declines in HIV among other subpopulations (general population, female sex workers), HIV among MSM has remained on a sustained increase globally $[1,3]$. In high income countries, the trend of HIV

\footnotetext{
* Correspondence: dreluwag@gmail.com

${ }^{1}$ Population Council, No. 16, Mafemi Crescent, Utako, Abuja, Nigeria Full list of author information is available at the end of the article
}

epidemic has been on a decline except among MSM [1]. Similarly, available data on HIV incidence and prevalence from low and middle-income countries suggest that the HIV epidemic among gay, bisexual and other men who have sex with men are on a markedly different and increasing trajectory [1-3]. In the USA, new HIV infections among MSM has been estimated to be increasing at $8 \%$ per annum since 2001 [1]. In the Amsterdam Cohort Study among MSM, HIV acquisition increased from 1.0 per 100 person-years in 1992 to 2.0 per 100-person years in $2009[4,5]$ while in China, from prospective cohort studies conducted among MSM between 2005 and 2007,

C The Author(s). 2019 Open Access This article is distributed under the terms of the Creative Commons Attribution 4.0 International License (http://creativecommons.org/licenses/by/4.0/), which permits unrestricted use, distribution, and reproduction in any medium, provided you give appropriate credit to the original author(s) and the source, provide a link to the Creative Commons license, and indicate if changes were made. The Creative Commons Public Domain Dedication waiver (http://creativecommons.org/publicdomain/zero/1.0/) applies to the data made available in this article, unless otherwise stated. 
HIV acquisition was reported to have increased from 2.6 to 5.6 per 100 person-years [6-9].

Most studies of HIV acquisition and transmission among MSM have largely focused on individual level risk factors including unprotected receptive anal intercourse, high number of lifetime male partners, injecting and non-injecting drug use and high viral load in the index partner [1]. However, individual level risk factors alone, have been shown to be insufficient to explain the high transmission dynamics of HIV among MSM and the divergence of MSM epidemics when compared to HIV epidemics in other populations $[1,2]$. Other risk factors such as biological, couple-network level, communitylevel and structural drivers have been established to be pertinent in understanding the persistent high transmission rates among MSM especially in the presence of increased ART coverage whereby new infections should decrease as a result of reduced likelihood of transmission because of the effect of ART on viral load [2, 10,11].

MSM in Nigeria are conservatively estimated to be less than $1 \%$ of the Nigerian population yet nationally account for about 20\% of new HIV infections [12]. MSM are criminalized and stigmatized and this has further worsened in recent years with the passing of the SameSex Marriage Prohibition law of 2014 [13-17]. The new law included clauses that prohibited organizations from providing services to MSM and facilitation of meetings that support gay people, thus further criminalizing same-sex activities [12]. Studies have shown that these restrictive policies further limit the poor coverage of HIV prevention, treatment, and care programs among MSM [12, 18-21]. Very few MSM-targeted HIV prevention, treatment and care services exist in a limited number of states in Nigeria. This is chiefly because of limited data on the size estimate of MSM across states as well as limited funding for key population dedicated programs. Data on MSM are largely from three rounds of the population-based Integrated Biological and Behavioural Surveillance Survey (IBBSS) conducted in, 2007, 2010, and 2014. This study aimed to assess the change in HIV prevalence and determine correlates of HIV among MSM in Nigeria. Evidence from this study will be used by policy maker and program managers for evidencebased decision making for HIV prevention among MSM in Nigeria.

\section{Methods}

\section{Study sites}

In 2007, only three states - Lagos, Kano and Cross River, were included in the IBBSS. In 2010, following increased awareness of the contribution of MSM to the HIV epidemic, an additional three states were included in the IBBSS - the Federal Capital Territory (FCT), Kaduna and Oyo, while in 2014, Enugu and Rivers states were added to the six states included in the 2010 IBBSS. The state selection ensured that five of the six geopolitical zones in Nigeria were represented in the survey. Thus, the south west (Lagos and Oyo), south east (Enugu), south south (Rivers), north central (FCT) and north west zones (Kano and Kaduna) contributed to the study.

\section{Sampling design and recruitment}

Each round of the IBBSS used respondent driven sampling given to the hidden nature of MSM and thus allows for comparison between and across rounds. Respondent driven sampling (RDS) has been described in detail in previous studies [22, 23]. Briefly, RDS is a modified chain referral non-random sampling method of recruitment that adjusts for the non-randomness using a mathematical model that weights each sample recruited [23]. Inclusion criteria was male aged 16 years and above with a history of oral and/or anal sexual contact with another man in the 6 months prior to the survey. Known MSM, designated as "seeds" began the chain of the referral network and use vouchers to recruit their peers into the study. Ten seeds were selected for each round and seeds were diversified by age, educational status and socioeconomic status. To avoid an over-representation of MSM with similar attributes, vouchers limited to three per recruit was used [23, 24]. In addition, to avoid repeat enrollment, only one screener was used, only one person was approved to reimburse MSM who had successfully recruited his peers and only one location was used. Each voucher was redeemable and yielded N500 [approx. $\$ 3 \mathrm{USD}]$ as an incentive for participating, with an additional N500 given to a recruit for each successful additional participant. Total compensation was limited to a maximum compensation of N2000 [approximately \$12 USD]. Sample size for the 2007 IBBSS was estimated based on an assumed HIV prevalence of $15 \%$, a design effect of 2.0 and level of precision of 0.05 . For the 2010 and 2014 IBBSS, sample size was estimated to detect a $10 \%$ change from the subsequent round.

\section{Data collection}

Structured close-ended interviewer administered questionnaires elicited information on socio-demographic characteristics, type of sex partners and sexual risk behaviors. Interviews were conducted in MSM friendly organizations identified in each of the study states. Transactional sex was assessed both with female and male partners. Type of anal sex practiced was categorized as "insertive penile sex" or "receptive penile sex" in the past 6 months. HIV risk perception was assessed by asking MSM "do you feel you are at risk of infection with HIV?" with response options being "yes or no". Consistent condom use with sexual partners during transactional and non-transactional sex was assessed by asking the questions "how often did you or your male 
partner use a condom every time you had sex in the last six months?" while condom use at last sex was assessed by the question "the last time you had anal sex did you or your partner use a condom?". Transactional sex was assessed by asking "have you received money or gift in exchange for sex in the last 6 months?" Written consent was obtained from all participants for both behavioural and biological components of the survey.

\section{Laboratory testing}

Detection of HIV during all the studies was consistently done by rapid test using whole blood samples obtained from a finger prick. Based on the national HIV testing guideline, a parallel algorithm of Determine (Alere Medical, USA) and Unigold (Trinity Biotech, Plc, Bray, Ireland) was used to identify HIV sero-positivity while status of discordant tests was confirmed with the use of Stat Pak (Chembio Diagnostic Systems, New York, USA).

\section{Data management and analysis}

Data from each study state were entered centrally using CS Pro version 3.2 and 25\% of questionnaires underwent double-data entry to ensure data quality. Behavioural and biological data were linked by study unique identification number for each participant. Data from each round were merged and analyzed using STATA 14.0 (STATA Corporation). Descriptive statistics of demographic, behavioural, and biological variables was conducted. The Cochran-Armitage Chi Square trend test was used to compare differences between categorical variables across different rounds of IBBSS, while the Kruskal-Wallis test was used to compare differences in continuous variables across different rounds of IBBSS. To measure associations between HIV and predictor variables (Tables), bivariate logistic regression analysis was conducted with $p$ value $\leq 0 \cdot 2$ designated as the cutoff for inclusion in multivariate logistic regression models. Variables were retained if they attained a $\mathrm{p}$ value $\leq 0.05$ in the multivariate analysis. The predictor variables were based on data from literature that showed an association between the variables and HIV.

\section{Results}

\section{Sociodemographics}

Table 1 highlights the sociodemographic data of MSM across the three rounds of the IBBSS. A total of 897, 1545 and 3611 MSM were surveyed in 2007, 2010 and 2014 respectively. Many of the respondents were aged 20-24 years in 2007 (61\%) and 2014 (46\%), while in 2010 , almost half $(47 \%)$ of the respondents were 25 years or older. Over $85 \%$ of the respondents had never married in each round of the survey. Majority of the respondents had completed at least secondary level education, and this was highest among respondents in 2007 with $63 \%$ followed by respondents in 2014 (55\%).

\section{Sexual risk Behaviours}

The median number of insertive anal partners was consistent between 2007 and 2010 with about 50\% of the respondents reporting insertive anal sex (IAS) with at least two partners interquartile range ([IQR] 1-4) in the last 6 months. For receptive anal sex (RAS), a median of two sexual partners was reported in 2007 while it was one partner in both 2010 and 2014. Overall, about a third had engaged in transactional sex in 2007 (95\% confidence interval (CI):30-36\%) and 2014 (32-35\%), while it was about two-fifths in 2010 (36-41\%). Consistent condom use, when sex was sold increased steadily from $35 \%$ (27-44\%) to 79\% (74-83\%) between 2007 and 2014 . Similarly, consistent condom use in the past 6 months prior to the survey during paid sexual encounters increased from $35 \%(30-41 \%)$ in 2007 to $61 \%(58-64 \%)$ in 2014 . For noncommercial partners, consistent condom use increased from $32 \%$ (29-36\%) in 2007 to 51\% (49-53\%) in 2014. For self-reported experiences of sexually transmitted infection (STI), there was an increase from 7\% (5-9\%) in 2007 to $15 \%(13-17 \%)$ in 2010 and 19\% (17-20\%) in 2014.

\section{HIV risk perception and exposure to HIV interventions}

Only about a third of respondents (32\%; 95\% CI:29-35\%) felt they were at risk to HIV in 2007. This further decreased to $28 \%(25-30 \%)$ in 2010 and remained at $28 \%$ $(26-29 \%)$ in 2014 with borderline significance $(p=0.055)$. Self-reported previous HIV test increased steadily from $34 \%(31-37 \%)$ in 2007 to $59 \%$ (56-61\%) in 2010 and 65\% (63-66\%) in 2014. An assessment of the recency of the HIV test showed that over $70 \%$ reported that they received their HIV test within 1 year of the study, with the highest recent tests reported in 2014 (79\%; 77-81\%).

\section{Change in HIV prevalence}

As shown in Table 1, HIV prevalence increased steadily between 2007 and 2014. From 14\% (11-16\%) in 2007, it increased to $17 \%(15-19 \%)$ in 2010 and $23 \%(22-34 \%)$ in 2014. Table 2 shows HIV prevalence disaggregated by risk behaviors. When analysis was restricted to only states that participated in the three rounds of the IBBSS (Cross River, Kano and Lagos) HIV prevalence was 13\% (11-16\%) in 2010 and 25\% (22-27\%) in 2014.

Among those who reported RAS only, HIV prevalence was $19 \%(14-26 \%)$ in $2007,23 \%(19-28 \%)$ in 2010 and $24 \%(22-28 \%)$ in 2014 . By age group, HIV prevalence was stable among those aged $16-19$ years $(p=0.953)$ while it increased among those aged 20-24 years, from $9 \%(7-12 \%)$ in 2007 to $21 \%(19-23 \%)$ in 2014.

For the six states with data from at least two rounds of IBBSS, there was increase in HIV prevalence in four of 
Table 1 Sociodemographics, HIV prevalence and sexual risk behaviors among MSM 2007-2010

\begin{tabular}{|c|c|c|c|c|c|c|c|}
\hline \multirow[t]{2}{*}{ Characteristics } & $2007(n=897)$ & $95 \% \mathrm{Cl}$ & $2010(n=1545)$ & $95 \% \mathrm{Cl}$ & $2014(n=3611)$ & $95 \% \mathrm{Cl}$ & $p$ value \\
\hline & \multicolumn{2}{|l|}{$\%(n)$} & \multicolumn{2}{|l|}{$\%(n)$} & \multicolumn{3}{|l|}{$\%(n)$} \\
\hline HIV prevalence & 13.5 (109) & $11.3-16.0$ & $17.2(222)$ & $15.2-19.4$ & $22.9(711)$ & $21.5-24.4$ & $<0.0001$ \\
\hline \multicolumn{8}{|l|}{ Age (yrs) } \\
\hline $16-19$ & $13.2(116)$ & $11.1-15.6$ & $13.8(213)$ & $12.2-15.6$ & $20.9(756)$ & $19.6-22.2$ & \\
\hline $20-24$ & $60.5(532)$ & $57.2-63.7$ & $39.6(612)$ & $37.2-42.1$ & 45.9 (1658) & $44.3-47.5$ & \\
\hline$\geq 25$ & $26.3(231)$ & $23.5-29.3$ & $46.6(720)$ & $41.1-49.1$ & 33.2 (1197) & $31.6-34.7$ & $<0.0001$ \\
\hline Ever married & $3.9(34)$ & $2.8-5.4$ & $12.3(189)$ & $10.7-14.0$ & $6.9(247)$ & $6.1-7.7$ & 0.596 \\
\hline \multicolumn{8}{|l|}{ Educational Level } \\
\hline None & $2.1(18)$ & $1.3-3.3$ & $3.7(57)$ & $2.9-4.8$ & $3.1(112)$ & $2.9-3.7$ & \\
\hline Primary & $19.2(168)$ & $16.7-22.0$ & 20.0 (309) & $18.1-22.1$ & $13.9(500)$ & $12.8-15.0$ & \\
\hline Secondary & $63.3(553)$ & $60.0-66.4$ & $50.3(777)$ & $47.8-52.8$ & $55.4(2000)$ & $53.8-57.0$ & \\
\hline Tertiary & 15.5 (135) & $13.2-18.0$ & $26.0(402)$ & 23.9-28.3 & 27.7 (999) & $26.2-29.2$ & $<0.0001$ \\
\hline \multicolumn{8}{|l|}{$\S$ Sexual partners in past 6 months } \\
\hline Median No. of insertive anal sex partners (IQR) & $2(1-4)$ & & $2(0-4)$ & & $2(0-3)$ & & 0.0001 \\
\hline Median No. of receptive anal sex partners (IQR) & $2(1-4)$ & & $1(0-3)$ & & $1(0-3)$ & & 0.0001 \\
\hline Median No. of partners who paid for sex (IQR) & $0(1-4)$ & & $0(0-2)$ & & $0(0-1)$ & & 0.0001 \\
\hline Median No. of non commercial sex partners (IQR) & $3(1-5)$ & & $1(0-3)$ & & $1(1-1)$ & & 0.0001 \\
\hline Had female sex partner & $15.2(134)$ & $13.0-17.8$ & $52.2(785)$ & $49.7-54.7$ & $72.4(2537)$ & $70.9-73.8$ & $<0.0001$ \\
\hline Engaged in transactional sex & $32.7(276)$ & $29.6-35.9$ & $38.8(555)$ & $36.3-41.3$ & $33.2(1130)$ & $31.6-34.8$ & 0.161 \\
\hline \multicolumn{8}{|l|}{ Consistent condom use during sex in the past 6 month } \\
\hline Consistent condom use when selling sex & $35.0(42)$ & $27.0-44.0$ & $52.1(96)$ & $44.9-59.3$ & $78.6(265)$ & 73.9-82.7 & $<0.0001$ \\
\hline Consistent condom use when buying sex & $35.2(95)$ & $29.7-41.1$ & $34.2(176)$ & $30.2-38.4$ & $61.3(669)$ & $58.4-64.2$ & $<0.0001$ \\
\hline $\begin{array}{l}\text { Consistent condom use with non-commercial } \\
\text { partners }\end{array}$ & $32.2(236)$ & $28.9-35.6$ & $34.9(320)$ & $31.8-38.0$ & $50.8(1406)$ & $48.9-52.6$ & $<0.0001$ \\
\hline \multicolumn{8}{|l|}{ Sexual position in past 6 months } \\
\hline Engaged in receptive sex only & $20.5(179)$ & $18.0-23.3$ & $27.7(423)$ & $25.5-30.0$ & $23.8(852)$ & $32.1-35.2$ & \\
\hline Engaged in insertive sex only & $24.4(213)$ & $21.7-27.4$ & $38.4(586)$ & $36.0-40.9$ & $33.7(1206)$ & $32.1-35.2$ & \\
\hline Engaged in both insertive and receptive sex & $55.1(480)$ & $51.7-58.3$ & $33.9(517)$ & $31.5-36.3$ & $42.6(1525)$ & $41.0-44.2$ & 0.002 \\
\hline \multicolumn{8}{|l|}{ HIV risk and exposure to interventions } \\
\hline Ever tested for HIV & $34.0(299)$ & $31.0-37.2$ & $58.6(777)$ & $55.9-61.2$ & $64.6(2312)$ & $63.0-66.2$ & $<0.0001$ \\
\hline Tested for HIV within 1 years of survey & $72.9(218)$ & $67.6-77.7$ & $77.1(533)$ & $73.9-80.1$ & $78.9(2327)$ & $77.2-80.6$ & 0.019 \\
\hline Feels at risk to HIV & $32.3(278)$ & $29.2-35.4$ & $27.8(322)$ & $25.3-30.4$ & $27.6(990)$ & $26.2-29.1$ & 0.055 \\
\hline Experienced an STI in the past 6 months & $6.8(60)$ & $5.3-8.7$ & $15.0(232)$ & $13.3-16.9$ & $18.6(670)$ & $17.3-19.9$ & 0.039 \\
\hline
\end{tabular}

\$Sexual position as defined by the sexual position (insertive, receptive or both) practiced by the respondent. $p$ values derived from Cochran-Armitage Chi-square trend test

the states between the two rounds, while two states recorded declines. For Cross River state, HIV prevalence increased from 3\% (1-5\%) in 2007 to 6\% (4-9\%) in 2010 and $11 \%(9-15 \%)$ in 2014 while in FCT $(44 \%$ [36-53\%] vs. $30 \%$; [26-34\%] and Kaduna state (23\% [18-29\%] vs. $16 \%[13-19 \%])$ there was about $30 \%$ decrease in HIV prevalence between 2010 and 2014 .

\section{Factors associated with HIV prevalence among MSM}

Table 3 outlines factors associated with HIV among MSM in Nigeria. When compared to MSM aged 16-19 years, those aged $20-24$ years and $\geq 25$ years were more likely to be HIV positive (adjusted odds ratio [AOR] 1.40; 95\% CI: 1.09-1.80) and (AOR 2.41; 95\% CI:1.84-3.16) respectively. Compared to those who engaged in IAS only, those who engaged in RAS only (AOR 1.68; 95\% CI:1.11-2.54) or both IAS and RAS (AOR 1.71; 95\% CI:1.40-2.10) were more likely to be HIV positive. With Cross River state as the reference, MSM in Enugu state (AOR 1.89; 95\% CI: 1.262.80), FCT (AOR:4.23; 95\% CI:3.04-5.87), Kaduna state (AOR: 2.27; 95\% CI: 1.59-3.23), Kano state (AOR: 1.97; 95\% CI: 1.29-3.00), Lagos state (AOR:6.66; 95\% CI: 4.93- 
Table 2 HIV prevalence disaggregated by sociodemographics and sexual risk behaviours

\begin{tabular}{|c|c|c|c|c|c|c|c|}
\hline Characteristics & $2007 \%(n)$ & $95 \% \mathrm{Cl}$ & $2010 \%(n)$ & $95 \% \mathrm{Cl}$ & $2014 \%(n)$ & $95 \% \mathrm{Cl}$ & $p$ value \\
\hline \multicolumn{8}{|l|}{ Age (years) } \\
\hline $16-19$ & $13.3(13)$ & $7.8-21.6$ & $12.0(24)$ & $8.2-17.3$ & $12.4(82)$ & $10.1-15.1$ & 0.909 \\
\hline $20-24$ & $8.9(44)$ & $6.7-11.7$ & $16.2(87)$ & $13.3-19.6$ & $21.1(303)$ & $19.1-23.3$ & $<0.0001$ \\
\hline$>=25$ & $24.1(52)$ & $18.8-30.2$ & $20.0(111)$ & $16.9-23.6$ & $32.3(326)$ & $29.5-35.3$ & $<0.0001$ \\
\hline Engaged in receptive sex only in past 6 months & $19.0(31)$ & $13.7-25.8$ & $23.1(76)$ & 18.9-28.0 & $24.4(187)$ & $21.5-27.6$ & 0.177 \\
\hline Engaged in insertive sex only in past 6 months & $11.3(23)$ & $7.6-16.5$ & $14.1(69)$ & $11.2-17.5$ & $18.6(193)$ & $16.3-21.1$ & 0.002 \\
\hline Engaged in both insertive and receptive sex in past 6 months & $12.4(54)$ & $9.6-15.8$ & $16.0(73)$ & $12.9-19.7$ & $25.1(321)$ & $22.8-27.6$ & $<0.0001$ \\
\hline Engaged in transactional sex in past 6 months & $14.6(37)$ & $10.7-19.5$ & $18.7(89)$ & $15.4-22.4$ & $18.7(191)$ & $16.5-21.3$ & 0.218 \\
\hline Feels at risk to HIV & $18.9(49)$ & $14.6-24.2$ & $24.1(60)$ & $19.2-29.8$ & $27.3(238)$ & $24.4-30.4$ & 0.006 \\
\hline Experienced STI symptoms in past 6 months & $10.5(6)$ & $4.8-21.7$ & $21.3(43)$ & $16.2-27.5$ & $25.0(151)$ & $21.7-28.7$ & 0.019 \\
\hline Had female sex partner in past 6 months & $10.2(13)$ & $6.0-16.9$ & $16.6(108)$ & $13.9-19.6$ & $23.3(494)$ & $21.5-25.1$ & $<0.0001$ \\
\hline \multicolumn{8}{|l|}{ States } \\
\hline Cross River & $2.8(8)$ & $1.4-5.4$ & $5.7(14)$ & $3.6-9.1$ & $11.3(52)$ & $8.7-14.5$ & $<0.0001$ \\
\hline Enugu & NA & & NA & & $16.8(69)$ & $13.5-20.8$ & \\
\hline FCT & NA & & $44.4(55)$ & $35.8-53.2$ & 30.1 (169) & $26.4-34.0$ & 0.002 \\
\hline Kaduna & NA & & $23.1(48)$ & $17.8-29.3$ & $15.5(76)$ & $12.5-19.0$ & 0.016 \\
\hline Kano & $11.7(27)$ & $8.2-16.6$ & $11.4(31)$ & $8.1-15.7$ & 14.9 (13) & $8.8-24.1$ & 0.514 \\
\hline Lagos & $25.4(74)$ & $20.7-30.8$ & $27.1(52)$ & $21.2-33.8$ & $41.4(172)$ & $36.7-46.2$ & $<0.0001$ \\
\hline Oуо & NA & & $9.6(19)$ & $6.2-14.6$ & $14.2(62)$ & $11.2-17.8$ & 0.105 \\
\hline Rivers & NA & & NA & & 40.7 (98) & $34.6-47.0$ & \\
\hline
\end{tabular}

$P$ value derived from Chocran_Armitage Chi-square trend tests. $C I$ Confidence interval

8.99) and Rivers state (AOR: 7.37; 95\% CI:4.96-10.94) were more likely to be HIV positive. Education and transactional sex were not associated with HIV among MSM in Nigeria.

\section{Discussion}

This is the first study to conduct a trend analysis of HIV prevalence and its correlates among MSM in Nigeria and we identified several important findings. First, HIV prevalence has steadily increased over time with a 10percentage point increase every year over 7 years. Second, the burden of HIV is higher among older MSM than younger ones. Third, prevalence of STI has also increased over the years and has more than doubled from $7 \%$ in 2010 to $17 \%$ in 2014. Fourth, although consistent condom use has increased with transactional sex, the increase is less with non-transactional sex. Fifth, less than $70 \%$ of MSM have ever been tested for HIV highlighting major gaps in HIV prevention intervention for MSM. Sixth, only about a third of MSM felt they were at risk for HIV and lastly, compared to Cross River states, MSM who reside in other states except Kano state were more likely to be HIV positive. These findings directly mirror the state of HIV programming for MSM in Nigeria and strategies, policies and programs must be designed to address these gaps.

Between the first and second rounds of IBBSS in 2007 through 2010, female sex workers (FSW) had shown the highest prevalence of HIV among key populations in Nigeria. However, in the third round of IBBSS in 2014, HIV prevalence among MSM (23\%) exceeded that of FSW (19\%) which has been on a decline from $37 \%$ to $19 \%$ and 30 to $9 \%$ among brothel and non-brothel based female sex workers respectively [25]. The relative increase in HIV incidence among MSM in the era of expanded ART and in which there's been HIV decline among other groups has been termed "resurgent epidemic in MSM" [1, 4, 26, 27] and future studies among this group may benefit from incidence studies to estimate the HIV incidence rate among MSM in Nigeria. Unprotected anal intercourse (UAI) remains the main risk factor for HIV among MSM and studies have demonstrated the high transmission efficiency of HIV through anal sex [1]. Kingsley et al. (1987) reported a 20 -fold increased risk of HIV seroconversion over 6 months among MSM who reported UAI when compared to those who did not $[4,28]$. Baggaley et al., (2008) in a systematic review and meta-analysis of HIV transmission risks in anal sex, reported a $1.4 \%$ transmission probability per-act of unprotective receptive anal intercourse (URAI) and 40.4\% (6.0-74.9) per-partner probability, with no difference between MSM and heterosexual anal intercourse [29]. The $1.4 \%$ per-act probability for URAI has been estimated to be roughly 18-times greater than that of vaginal intercourse [1,30]. An updated review in 2018, showed a pooled HIV-1 risk of 1.3\% 
Table 3 Multivariate analysis showing factors associated with HIV among MSM in Nigeria

\begin{tabular}{|c|c|c|c|c|}
\hline Factors & Crude OR (95\% Cl) & $p$ value & Adjusted OR (95\% Cl) & $p$ value \\
\hline \multicolumn{5}{|c|}{ Age (years) } \\
\hline $16-19$ & 1 & & 1 & \\
\hline $20-24$ & $1.51(1.21-1 \cdot 88)$ & $<0.0001$ & $1.40(1.09-1 \cdot 80)$ & 0.008 \\
\hline$>=25$ & $2.68(2.15-3.33)$ & $<0.0001$ & $2.41(1.84-3 \cdot 16)$ & $<0.0001$ \\
\hline
\end{tabular}

Ever married

$\begin{array}{lll}\text { No } & 1 & \\ \text { Yes } & 0.97(0.75-1.26) & 0.817\end{array}$

Educational level

$\begin{array}{ll}\text { None } & 1 \\ \text { Primary } & 0.6(0.40-0.91) \\ \text { Secondary } & 0.79(0.54-1.15) \\ \text { Tertiary } & 1.25(0.85-1.84) \\ \text { Sexual position in past 6 months } & \\ \text { Insertive sex only } & 1 \\ \text { Receptive only } & 1.55(1.29-1.86) \\ \text { Both } & 1.32(1.12-1.56)\end{array}$

Engaged in transactional sex in past 6 months

$\begin{array}{ll}\text { No } & 1 \\ \text { Yes } & 0.86(0.74-0.99)\end{array}$

817

consistent condom when paid for sex in past 6 months

\section{Always}

Sometimes

\section{1}

$0.76(0.42-1 \cdot 36)$

Feels at risk to HIV

$\begin{array}{ll}\text { No } & 1 \\ \text { Yes } & 1.06(1.01-1.12) \\ \text { Don't know } & \end{array}$

Don't know

Experienced STI symptoms in past 6 months

$\begin{array}{ll}\text { No } & 1 \\ \text { Yes } & 1.26(1.05-1.50)\end{array}$

Had female sexual partner in past 6 months

$\begin{array}{ll}\text { No } & 1 \\ \text { Yes } & 1.23(1.07-1.42) \\ \text { States } & \\ \text { Cross River } & 1 \\ \text { Enugu } & 2.54(1.80-3.60) \\ \text { FCT } & 6.10(4.60-8.08) \\ \text { Kaduna } & 2.71(2.00-3.67) \\ \text { Kano } & 1.72(1.22-2.42) \\ \text { Lagos } & 6.23(4.76-8.17) \\ \text { Oyo } & 1.84(1.32-2.56) \\ \text { Rivers } & 8.62(6.09-12.18) \\ \text { Year } & \\ 2007 & 1 \\ 2010 & 1.34(1.04-1.71) \\ 2014 & 1.91(1.54-2.38)\end{array}$

0.015

0.218

0.255

$0.87(0.53-1.42)$

0.571

$1.02(0.65-1.61)$

0.917

$1.16(0.73-1.84)$

0.534

$<0.0001$

$1.92(1.54-2.40)$

$<0.0001$

0.001

$1.71(1.40-2.10)$

$<0.001$

1

$0.91(0.77-1.09)$

0.308
$1.36(1.14-1.61)$

$0.96(0.59-1.54)$

1

$1.26(1.02-1.55)$

0.034

1

$0.93(0.77-1 \cdot 12)$

0.439

1

$<0.0001$

$<0.0001$

$<0.0001$

$<0.002$

$<0.0001$

$<0.0001$

$<0.0001$

$1.89(1.26-2 \cdot 80)$

$4.23(3.04-5.87)$

$2.27(1.59-3.23)$

$1.97(1.29-3.00)$

6.66 (4.93-8.99)

$1.65(1.13-2.39)$

7.37 (4.96-10.94)

0.002

$<0.0001$

$<0.0001$

0.002

$<0.0001$

0.009

$<0.0001$

1

0.022

$<0.0001$
$1.92(1.40-2.62)$

$2.04(1.51-2.75)$
$<0.0001$

$<0.0001$

OR Odds ratio, $\mathrm{Cl}$ Confidence interval 
for URAI with no difference between the pre-ART and ART era ( $1.7 \%$ vs. $0.8 \% ; p=0.537)$ [31]. Findings from our study showed that those who engaged in receptive anal sex only, were twice more likely to be HIV positive compared to those who reported only insertive anal sex. Similarly, those who engaged in both insertive and receptive anal sex were twice as likely to be HIV positive when compared to only those who practiced insertive anal sex. This corroborates Baggaley et al's (2008) findings in their systematic review where per-partner risks for infection were similar for people reporting exclusive unprotected receptive anal intercourse and both unprotected receptive and insertive anal intercourse $[1,29]$. These factors have been suggested as key drivers of the rapid and efficient spread of HIV through networks of MSM [2].

Consistent condom use provides about $70-80 \%$ efficacy in preventing HIV transmission [2, 32-34]. In this study, consistent condom use increased from 2007 to 2014, when sex was sold or bought and with non-transactional partners. However, while consistent condom use in past 6 months more than doubled when sex was sold, only $50 \%$ of those who reported non-transactional sex used condoms consistently. This suggests that within MSM sexual networks, there's an increased probability of HIV transmission which may negate the increased use of condom during transactional sex. Sero-adaptation, including serosorting and strategic or sero-positioning, which rely on knowing one's HIV status as well as that of their sex partners, have been used by MSM as prevention approaches $[1,35]$. Serosorting involves the selection of HIV-concordant sex partners, while sero-positioning involves choosing sex acts based on serostatus [1]. A study in Seattle, U.S.A, showed that among recently infected MSM, 69\% reported UAI with HIV-positive or unknown status partners compared with $32 \%$ in HIV uninfected controls $[4,36]$. The Swiss HIV cohort study reported that the strongest predictor of UAI was knowing the HIV status of sexual partners with consistent condom use being $89 \%$ between stable discordant couples and $48 \%$ between HIV-infected partners $[4,37]$. The role of seroadapation in Nigeria is unknown and more so with less than 70\% of MSM ever being tested for HIV, it's unlikely that this practice is widespread as the knowledge of HIV status of partners remains limited within networks.

The low consistent condom use in non-transactional sex may explain the significant increase in self-reported STI between 2007 and 2014. Furthermore, among those who reported STIs, HIV prevalence increased between 2007 and 2014. Higher prevalence of STIs and undiagnosed HIV infections are markers of suboptimal access to clinically competent and appropriate health care services which are in turn reported to reduce HIV-related health-seeking behaviour in African MSM $[1,38]$. The suboptimal access to healthcare and discrimination by healthcare workers are further worsened by the poor funding of MSM targeted prevention and treatment services in Nigeria. Between 2007 and 2012, less than 5\% of HIV funding was dedicated to most-at-risk population and less than $5 \%$ dedicated to enabling environment for HIV programs [39-41].

The increase in consistent condom use observed during transactional sex may explain the low perceived risk of HIV among MSM. Less than a third of MSM felt at risk of HIV and given the increased prevalence of HIV and STIs among MSM in Nigeria, efforts must be made to heighten HIV risk perception. The psychometric paradigm theory and a number of other social and health psychology theories [42-49] have identified risk perception as having a central role in determining behavior. A meta-analysis of risk appraisal reported that interventions that successfully heightened the risk appraisal within an individual, resulted to changes in subsequent intentions and behaviour [50]. Similarly, de Hoog et al. (2007) reported that when the severity of a threat was heightened, irrespective of the channel of communication, there was an associated positive and significant effect on intention and behaviour change [51]. Behaviour change interventions for HIV programs should be designed to heighten the threat of HIV.

Older MSM were more likely to be HIV positive than younger MSM. HIV prevalence among MSM aged 1619 years remained unchanged between 2007 and 2014 and was lower than that reported for those aged 20-24 years and those 25 years and above. Merrigan et al. (2010) reported similar results among MSM in three states in Nigeria [52]. Another recent study in Nigeria among MSM who engaged in transactional sex, showed that MSM aged 25 years and above were four times more likely to be HIV positive than those aged 15-19 years [53]. However, our findings are contrary to those reported by Beyrer et al. (2012) and (2016) which indicated that younger MSM had higher burden of HIV [1, 54]. A plausible explanation for our finding is that the older MSM have had prolonged exposure to HIV through higher number of sexual partners, engaging in transactional sex and higher exposure to unprotected anal sex. Furthermore, MSM sampled in 2010 and 2014 were more likely to be HIV positive compared to those in 2007 and this further supports our argument that the prolonged exposure to higher risk behaviours may be the reason behind higher HIV prevalence among older MSM. Younger MSM aged 16-19 years are likely to still have parental support and thus, there's less socioeconomic pressure to engage in high risk sexual practices such as transactional sex. In addition, their sexual networks revolve around their peers rather than intergenerational sexual partners and this limits their exposure to older HIV infected MSM. 
There was a significant increase in the proportion of MSM who reported having sex with female partners between 2007 and 2014. This constitutes a potential bridge between MSM and the general population and thus merits discussion as the gains in reduction of HIV prevalence among the general population may be eroded by bisexual intercourse among MSM. The increase in bisexuality may reflect the increasing hostility, stigma and criminalization of MSM in Nigeria. Schwartz et al. (2015) assessed the immediate effects of the same-sex marriage prohibition act in Nigeria and reported a statistically significant increase in proportion of MSM who had female partners after the law was passed when compared to the pre-law period [13]. This coping mechanism to the high stigma and criminalization of MSM may also negatively impact their utilization of key population friendly clinics as they continue to hide their identity even to health care workers.

This study has some limitations. The absence of a prospective study group and the use of cross-sectional surveys from unmatched cohort limits the strength of our study and thus requires caution in the interpretation of the data. There may be potential dependence between data from different rounds of IBBSS which may overestimate HIV prevalence if a significant number of positives from previous rounds were recruited into subsequent rounds or an underestimation of HIV prevalence is a significant number of HIV negative MSM were targeted and recruited in subsequent rounds. Future studies should include a variable to help identify those in previous rounds and their HIV status at that round to allow a more robust estimation of HIV among MSM. Data on HIV prevention programs and treatment coverage in the study states was not available and thus could not be accounted for in our study to independently measure the impacts of these programs in the study outcome. In addition, data on treatment coverage could help explain the observed increase if treatment coverage was assessed to be low. Another limitation is that of social desirability bias on sexual risk behaviours as information were self-reported, however the higher increase in consistent condom use during transactional sex compared to nontransactional is comparable to that observed among female sex workers [54] and suggests that risks behaviors captured in these studies may have been under-reported given the increase of STIs and HIV observed. Furthermore, studies on biological validation of unprotected sex among female sex workers have shown significant over-reporting of protected sex [55] and future studies should consider biological validation of protected sex among MSM to better characterize risk behaviours Drug use especially use of methamphetamine [1] has been associated with HIV among MSM, however, there was no data on drug use among MSM in all three rounds of the survey. Further research is required to determine the association of drug use and HIV among MSM in Nigeria. Lastly, not all clients opted for an HIV test and the proportion of refusal ranged from 10 to $16 \%$. While the status of those who rejected an HIV test cannot be assumed, participants who refused to opt for an HIV test may have done so because of previous knowledge of HIV infection and thus prevalence of HIV may have been underestimated in the current study and subsequently biases the observed trend in HIV prevalence.

\section{Conclusions}

In conclusion, this the first study to evaluate the trend of HIV prevalence among MSM in Nigeria and we report a number of key observations. As in other climes, HIV prevalence among MSM in Nigeria is on an alarming progression with a relative increase of $10 \%$ point per year over 7 years. No state is spared, and prevention packages must be holistic and involve the use of strategies with the strongest evidence of highest efficacy in preventing HIV transmission; early treatment of partners, [2, 56] condoms [32, 57] and oral preexposure prophylaxis [58]. Lastly, the HIV epidemic among MSM in Nigeria is severe and clearly, is one of the defining challenges ahead, and maybe the most critical gap in the national HIV prevention program to control the HIV epidemic in Nigeria.

\section{Abbreviations \\ AOR: Adjusted Odds Ratio; ART: Antiretroviral Treatment; Cl: Confidence Interval; FCT: Federal Capital Territory; FSW: Female Sex Workers; HIV: Human Immunodeficiency Virus; IAS: Insertive Anal Sex; IBBSS: Integrated Biological and Behavioral Surveillance Survey; MSM: Men who have sex with men; RAS: Receptive Anal Sex; RDS: Respondent Driven Sampling; STI: Sexually Transmitted Infections; UAl: Unprotected Anal Intercourse; URAI: Unprotected Receptive Anal Intercourse; USD: United States Dollars}

\section{Acknowledgements}

We would like to acknowledge members of the MSM community that availed their time and interest in participating in the different rounds of the IBBSS.

\section{Authors' contributions}

GIE conceived the study. GIE and SBA coordinated data collection. GIE, TE and SBA conducted data analysis. GIE, OO, OI and SBA interpreted the data. $\mathrm{GIE}, \mathrm{OO}$ and $\mathrm{OI}$ wrote the manuscript. SBA, TE, CN reviewed and revised the manuscript. All authors approved the final manuscript.

Funding

There was no funding for this study.

Availability of data and materials

Data are available under reasonable request from the corresponding author.

Ethics approval and consent to participate

Ethical approval was obtained from the National Health Ethics Research Committee (NHREC), Federal Ministry of Health. Written consent was obtained from all participants.

Consent for publication

Not applicable.

Competing interests

The authors declare that they have no competing interests. 


\section{Author details}

${ }^{1}$ Population Council, No. 16, Mafemi Crescent, Utako, Abuja, Nigeria. ${ }^{2}$ Save the Children International, Abuja, Nigeria. ${ }^{3}$ Centers for Disease Prevention and Control, United States Embassy, Abuja, Plot 1038 Diplomatic Drive, Abuja, Nigeria. ${ }^{4} \mathrm{HIV}$ and Viral Hepatitis Communicable Diseases Cluster, World Health Organization Country Office, Abuja, Nigeria. ${ }^{5}$ National AIDS and STI Control Program, Federal Ministry of Health, Abuja, Nigeria.

\section{Received: 15 January 2019 Accepted: 22 August 2019}

\section{Published online: 02 September 2019}

\section{References}

1. Beyrer C, Baral SD, van Griensven F, et al. Global epidemiology of HIV infection in men who have sex with men. Lancet. 2012;380:367-77.

2. Beyrer C, Sullivan P, Sanchez J, et al. The increase in global HIV epidemics in MSM. AIDS. 2013;27:2665-78

3. Centers for Disease Control and Prevention (CDC). HIV infections attributed to male-to-male sexual contact - metropolitan statistical areas, United States and Puerto Rico, 2010. MMWR Morb Mortal Wkly Rep. 2012;61:962-6.

4. Morris SR, Little SJ. MSM: resurgent epidemics. Curr Opn HIV AIDS. 2011;6: 326-32.

5. Jansen IA, Geskus RB, Davidovich U, et al. Ongoing HIV-1 transmission among msn who have sex with men in Amsterdam: a 25-year prospective cohort study. AIDS. 2011:25:493-501.

6. van Griensven F, Varangrat A, Wimonsate W, et al. Trends in HIV prevalence, estimated HIV incidence, and risk behavior among men who have sex with men in Bangkok, Thailand, 2003-2007. J Acquir Immune Defic Syndr. 2009; 53:234-9.

7. Ruan $Y$, Jia $Y$, Zhang $X$, et al. Incidence of HIV-1, syphilis, hepatitis $B$, and hepatitis $C$ virus infections and predictors associated with retention in a $12-$ month follow-up study among men who have sex with men in Beijing, China. J Acquir Immune Defic Syndr. 2009;52:604-10.

8. Yang $\mathrm{H}, \mathrm{Hao} \mathrm{C}$, Huan $\mathrm{X}$, et al. HIV incidence and associated factors in a cohort of men who have sex with men in Nanjing, China. Sex Transm Dis. 2010;37:208-13.

9. Xu JJ, Zhang M, Brown K, et al. Syphilis and HIV seroconversion among a 12-month prospective cohort of men who have sex with men in Shenyang, China. Sex Transm Dis. 2010;37:432-9.

10. Das M, Chu PL, Santos G, et al. Decreases in community viral load are accompanied by reductions in new HIV infections in San Francisco. PLoS One. 2010. https://doi.org/10.1371/journal.pone.0011068.

11. Charlebois ED, Das M, Porco TC, Havlir DV. The effect of expanded antiretroviral treatment strategies on the HIV epidemic among men who have sex with men in San Francisco. Clin Infect Dis. 2011:52:1046-9.

12. Schwarts SR, Nowak R, Orazulike I, et al. The immediate effect of the samesex marriage prohibition act on stigma, discrimination, and engagement on HIV prevention and treatment services in men who have sex with men in Nigeria: analysis of prospective data from the TRUST cohort. Lancet. 2015. https://doi.org/10.1016/s2352-3018(15)00078-8.

13. Goitom H. Nigeria: bill prohibiting same-sex marriage moves forward. 2013. http://www.loc.gov/lawweb/servlet/lloc_news?disp3_I205403807_text. Accessed 11 Sept 2017.

14. Gladstone R. Nigerian President signs ban on same-sex relationships. 2014 http://www.nytimes.com/2014/01/14/world/africa/nigerian-president-signsban-on-same-sexrelationships.html. Accessed 11 Sept 2017.

15. Itaborahy LP, Zhu J. A world survey of laws: criminalisation, protection and recognition of same-sex love. Geneva: International Lesbian Gay Bisexual Trans and Intersex Association; 2014.

16. National Assembly of the Federal Republic of Nigeria. Same Sex Marriage (Prohibition) Act, 2013. 2014. http://www.nassnig.org/nass/acts.php. Accessed 11 Sept 2017.

17. Semugoma P, Beyrer C, Baral S. Assessing the eff ects of antihomosexuality legislation in Uganda on HIV prevention, treatment, and care services. SAHARA J. 2012:9:173-6.

18. Poteat T, Diouf D, Drame FM, et al. HIV risk among MSM in Senegal: a qualitative rapid assessment of the impact of enforcing laws that criminalize same sex practices. PLoS One. 2011;6:e28760.

19. Baral S, Holland CE, Shannon K, et al. Enhancing benefits or increasing harms: community responses for HIV among men who have sex with men, transgender women, female sex workers, and people who inject drugs. J Acquir Immune Defi c Syndr. 2014;66(suppl 3):S319-28.
20. Gruskin S, Ferguson L, Alfven T, Rugg D, Peersman G. Identifying structura barriers to an effective HIV response: using the National Composite Policy Index data to evaluate the human rights, legal and policy environment. J Int AIDS Soc. 2013;16:18000.

21. Eluwa GI, Strathdee SA, Adebayo SB, Ahonsi B, Adebajo SB. A profile on HIV prevalence and risk behaviors among injecting drug users in Nigeria: should we be alarmed? Drug Alcohol Depend. 2013;127:65-71.

22. Eluwa Gl, Sylvia A, Luchters S, Ahonsi B. HIV risk perception and risk behaviors among men who have sex with men in Nigeria. J AIDS Clin Res. 2015;6:478. https://doi.org/10.4172/2155-6113.1000478.

23. Heckathorn DD, Semaan S, Broadhead RS, Hughes JJ. Extensions of respondents-driven sampling: a new approach to the study of injection drug users aged 18-25. AIDS Behav. 2002;6:55-67.

24. Federal Ministry of Health. Integrated biological and behavioural surveillance survey. Abuja: Federal Ministry of Health; 2014.

25. Centers for Disease Control and Prevention (CDC). Prevalence and awareness of HIV infection among men who have sex with men - 21 cities, United States, 2008. MMMWR Morb Mortal Wkly Rep. 2010;59:1201-7.

26. Sullivan $\mathrm{P}$, Hamouda $\mathrm{O}$, Delpech $\mathrm{V}$, et al. Reemergence of the HIV epidemic among men who have sex with men in North America, western Europe, and Australia, 1996-2005. Ann Epidemiol. 2009;19:423-31.

27. Kingsley LA, Detels $R$, Kaslow $R$, et al. Risk factors for seroconversion to human immunodeficiency virus among male homosexuals. Results from the Multicenter AIDS Cohot Study. Lancet. 1987;1:345-9.

28. Baggaley RF, White RG, Boily MC. HIV transmission risk through anal intercourse: systematic review, meta-analysis and implications for HIV prevention. Int J Epidemio. 2010;39:1048-63.

29. Grulich A, Zablostka I. Commentary: probability of HIV transmission through anal intercourse. Int J Epidemiol. 2010;39(4):1064-5. https://doi.org/10.1093/ ije/dyq101.

30. Baggaley RF, Owen BN, Silhol R, Elmes J, Anton P, McGowan I, et al. Does per-act HIV-1 transmission through anal sex vary by gender? An updated systematic review and meta-analysis. Am J Reprod Immunol. 2018. https:// doi.org/10.1111/aji.13039.

31. Smith $D$, Herbst JH, Zhang H, Rose C. Condom efficacy by consistency of use among men who have sex with men: US. Atlanta: CROI 2013; 2013.

32. Weller SC, Davis-Beaty K. Condom effectiveness in reducing heterosexual HIV transmission. Cochrane Database Syst Rev. 2002;(1):CD003255.

33. Vittinghoff E, Douglas J, Judson F, Mckirnan D, MacQueen K, Buchbinder SP. Per-contact risk of human immunodeficiency virus transmission between male sexual partners. Am J Epidemiol. 1999;150:306-11.

34. McFarland W, Chen YH, Raymond HF, et al. HIV seroadaptation among individuals, within sexual dyads, and by sexual episodes, men who have sex with men, San Francisco, 2008. AIDS Care. 2011;23:261-8

35. Thiede H, Jenkins RA, Carey JW, et al. Determinants of recent HIV infection among Seattle-area men who have sex with men. Am J Public Health. 2009; 99:S157-64.

36. Hasse B, Ledergerber B, Hirschel B, et al. Frequency and determinants of unprotected sex among HIV-infected persons: the Swiss HIV cohort study. Clin Infect Dis. 2010;51:1314-22.

37. White House Officer of National AIDS Policy. National HIV/AIDS strategy for the United States. Washington, DC: US Government; 2011.

38. National Agency for the Control of AIDS (NACA). National AIDS Spending Assessment 2007-2008. Abuja: National Agency for the Control of AIDS; 2009. Accessed 18 Sept 2017

39. National Agency for the Control of AIDS (NACA). National AIDS Spending Assessment 2009-2010. Abuja: National Agency for the Control of AIDS; 2011. Accessed 18 Sept 2017

40. National Agency for the Control of AIDS (NACA). National AIDS Spending Assessment 2011-2012. Abuja: National Agency for the Control of AIDS; 2013. Accessed 18 Sept 2017

41. Slovic P. Perception of risk: reflections on the psychometric paradigm. In: Krimsky S, Godling D, editors. Social theories of risk. Wesport: Praeger; 1992. p. 117-52.

42. Rosenstock IM. Historical origins of the health belief model. Health Educ Monogr. 1974;15:328-35

43. Leventhal $\mathrm{H}$. Findings and theory in the study of fear communications. Adv Exp Soc Psychol. 1970;5:119-86.

44. Rogers RW. Cognitive and physiological processes in fear appeals and attitude change: a revised theory of protection motivation. In: Cacioppo J, Petty R, editors. Social Psychophysiology. New York: Guilford Press; 1983. p. 153-77. 
45. Weinstein ND. The precaution adoption process. Health Psychol. 1988;7: $355-86$.

46. Witte K. Putting the fear back into fear appeals: the extended parallel process model. Commun Monogr. 1992:59:329-49.

47. Gibbons FX, Gerrard M, Blanton H, Russell DW. Reasoned action and social reaction: willingness and intention as independent predictors of health risk. J Pers Soc Psychol. 1998;74:1164-80.

48. Wagenaar BH, Sullivan PS, Stephenson R. HIV knowledge and associated factors among internet-using men who have sex with men (MSM) in South Africa and the United States. PLoS One. 2012;7:e32915.

49. Sheeran P, Harris PR, Epton T. Does heightening risk appraisals change people's intentions and behavior? A meta-analysis of experimental studies. Psychol Bull. 2014;140:511-43.

50. de Hoog N, Stroebe W, de Wit JBF. The impact of vulnerability to and severity of a health risk on processing and acceptance of fear-arousing communications: a meta-analysis. Rev Gen Psychol. 2007;11:258-85.

51. Merrigan M, Azeez A, Afolabi B, et al. HIV prevalence and risk behaviours among men having sex with men in Nigeria. Sex Transm Infect. 2010. https://doi.org/10.1136/sti.2008.034991.

52. Bamboye E, Badru T, Bamboye A. Transactional sex between men and its implications on HIV and sexually transmitted infections in Nigeria. J Sex Trans Dis. 2017. https://doi.org/10.1155/2017/1810346.

53. Beyrer C, Baral S, Collins C, et al. The global response to HIV in men who have with men. Lancet. 2016;388:198-206.

54. Eluwa GI, Strathedee SA, Adebajo SB, et al. Sexual risk behaviours and HIV among female sex workers in Nigeria. J Acquir Immune Defic Syndr. 2012; 61:507-14.

55. Giguère K, Béhanzin L, Guédou FA, et al. Biological Validation of SelfReported Unprotected Sex and Comparison of Underreporting Over Two Different Recall Periods Among Female Sex Workers in Benin Open Forum Infectious Diseases; 2019. https://doi.org/10.1093/ofid/ofz010.

56. Cohen MS, Chen YQ, McCauley M, et al. Prevention of HIV-1 infection with early antiretroviral therapy. N Engl J Med. 2011;365:493-505.

57. Sith $\mathrm{D}$, Herbst $\mathrm{JH}$, Zhang $\mathrm{H}$, et al. Condom efficacy by consistency of use among men who have sex with men. Atlanta: US CROl; 2013.

58. Grant RM, Lama JR, Anderson PL, et al. Prexposure chemoprohylaxis for HIV prevention in men who have sex with men. N Engl J Med. 2010;363:2587-99.

\section{Publisher's Note}

Springer Nature remains neutral with regard to jurisdictional claims in published maps and institutional affiliations.

Ready to submit your research? Choose BMC and benefit from:

- fast, convenient online submission

- thorough peer review by experienced researchers in your field

- rapid publication on acceptance

- support for research data, including large and complex data types

- gold Open Access which fosters wider collaboration and increased citations

- maximum visibility for your research: over $100 \mathrm{M}$ website views per year

At BMC, research is always in progress.

Learn more biomedcentral.com/submissions 